\title{
A utilidade dos indicadores da qualidade no gerenciamento de laboratórios clínicos
}

\section{Usefulness of quality indicators in the management of clinical laboratories}

Keila Furtado Vieira'; Edson Shusaku Shitara²; Maria Elizabete Mendes ${ }^{3}$; Nairo Massakazu Sumita ${ }^{4}$

unitermos
Laboratório clínico
Indicadores da qualidade
Benchmarking
Gestão laboratorial
Acreditação e certificação
Garantia da qualidade

\section{resumo}

O uso dos indicadores da qualidade vem sendo valorizado na gestão dos laboratórios clínicos para otimizar a qualificação e a quantificação das falhas nos diferentes processos laboratoriais, bem como para auxiliar a implantação de medidas corretivas e preventivas e apontar a eficácia das ações tomadas. O objetivo deste trabalho é discorrer sobre a evolução da qualidade na área da saúde, com ênfase na área laboratorial. Alguns indicadores laboratoriais citados na literatura nas fases pré-analítica, analítica e pós-analítica também são apresentados e discutidos neste artigo. Por fim, destaca-se a experiência brasileira do Programa de Indicadores Laboratoriais desenvolvido pela Sociedade Brasileira de Patologia Clínica/Medicina Laboratorial (SBPC/ML) em parceria com a Control-Lab e o projeto Model of Quality Indicator, em fase de desenvolvimento pela International Federation of Clinical Chemistry and Laboratory Medicine (IFCC).
The use of quality indicators has been appreciated in laboratory management so as to optimize quality and error quantification in several laboratory processes. Furthermore, it assists in the implementation of preventive and corrective measures and it shows their corresponding efficiency. The objective of the present study is to discuss the evolution of quality, mainly in the laboratory area, focusing on the importance of quality indicators in laboratory management. Some pre-analytical, analytical and post-analytical laboratory indicators are also presented and discussed in this work. Finally, we highlight the Brazilian initiative in the Laboratory Indicator Program developed by the Brazilian Society of Clinical Pathology and Laboratory Medicine (SBPC/ML) in partnership with Control-Lab and the Model of Quality Indicator project, which has been developed by the International Federation of Clinical Chemistry and Laboratory Medicine (IFCC). key words

Clinical laboratory

Laboratory quality

indicator

Benchmarking

Laboratory management

Accreditation and

certification

Quality assurance

1. Farmacêutica bioquímica; mestranda da Faculdade de Medicina da Universidade de São Paulo (FMUSP); Coordenadora técnica do Setor de Imunoquímica do Laboratório Clínico da UNIMED Sorocaba.

2. Hematologista/hemoterapeuta e patologista clínico; mestre em Ciências Médicas pela Universidade Estadual de Campinas (UNICAMP); coordenador médico do Laboratório Clínico da UNIMED Sorocaba.

3. Doutora em Medicina (Patologia); médica patologista clínica; chefe da Seção Técnica de Bioquímica de Sangue da Divisão de Laboratório Central (DLC) do Hospital das Clínicas (HC) da FMUSP (LIM-03 da Patologia Clínica); coordenadora do Núcleo de Qualidade e Sustentabilidade da DLC/HC-FMUSP.

4. Doutor em Medicina; professor da disciplina de Patologia Clínica da FMUSP; médico patologista clínico; diretor do Serviço de Bioquímica Clínica da DLC/HC-FMUSP (LIM-03 da Patologia Clínica); assessor médico em Bioquímica Clínica do Fleury Medicina e Saúde. 


\section{Introdução}

\section{Aspectos históricos}

O conceito de qualidade passou a ter grande destaque em função do desenvolvimento tecnológico observado no último século.

A história da qualidade tem início com a evolução industrial nos anos 1920, principalmente das indústrias bélicas, as quais, em decorrência da grande guerra mundial, necessitavam aumentar a produção de armamentos. Assim, surge a atividade de inspeção com finalidade de avaliar o produto final e separar os defeituosos, evitando sua comercialização. Esta constitui a primeira fase de evolução da qualidade, com a criação do departamento de engenharia de produção nas indústrias. Instrumentos estatísticos voltados para a medição e o controle da qualidade são usados para análise do produto final. W. Shewart, em 1931, publicou a obra intitulada Economic control of quality manufactured product, embasando cientificamente esses conceitos.

Em uma segunda fase, surge a preocupação com a qualidade em todos os processos de produção, admitindo-se que o grau de variabilidade do produto é devido às variações nas matérias-primas e máquinas utilizadas e ao operador destas. Tem início o controle estatístico por amostragem, com técnicas de limite de variação aceitável durante todo o processo fabril, não se restringindo apenas ao produto final. Nos anos 1940, o controle da qualidade torna-se disciplina acadêmica nos cursos de engenharia.

Nas décadas subsequentes, a evolução da qualidade tornou-se mais evidenciada, com destaque para o Japão, devido à necessidade de reconstrução econômica no pósguerra. Inicia-se a fase da garantia da qualidade, com o objetivo principal de prevenção. A preocupação com a qualidade chega ao gerenciamento das empresas. Em 1950, W. Edwards Deming cria um novo conceito em qualidade denominado ciclo PDCA, cujas inicias, em inglês, significam plan, do, check e act, ou planejar, executar, verificar e atuar corretivamente. No ano seguinte, Joseph M. Juran publica uma obra denominada Quality control handbook, cujo conteúdo contempla o controle de custos da qualidade, em que os termos custo da não qualidade e retrabalho são abordados. A. Feigenbaum, em 1956, propõe o conceito de controle total da qualidade, preconizando que a responsabilidade da qualidade do produto é de toda a organização e não somente do departamento de controle da qualidade.
Atualmente, as exigências da qualidade são tidas como atributos essenciais à sobrevivência das organizações no mercado, com ênfase na satisfação do cliente.

O fácil acesso às informações e a criação de órgãos de defesa do consumidor fizeram surgir um novo tipo de cliente, com perfil mais exigente e conhecedor de seu papel como consumidor, evidenciando que o conhecimento das necessidades do cliente é fundamental para sua fidelização.

Além disso, as empresas passaram a descobrir mais rapidamente o que seus concorrentes estavam fazendo de melhor. Essas descobertas ocorreram por meio da prática de benchmarking ou técnica de referenciação, iniciada pela empresa Xerox, no final da década de 1970, quando da suspeita de que o custo da produção havia sido maior que sua principal concorrente japonesa. Benchmarking é um processo contínuo de medidas de produtos, serviços e práticas para comparação com os competidores de mercado ou companhias reconhecidas como líderes no ramo ${ }^{(11)}$. Desse modo, a necessidade de busca contínua da qualidade, o aumento da produtividade e a redução de custos tornaramse essenciais para uma empresa manter-se no mercado e ser competitiva ${ }^{(1,5,12,13)}$.

\section{Qualidade na saúde}

$\mathrm{Na}$ área da saúde, a filosofia da qualidade não difere da aplicada nas indústrias. A adequação do produto ou serviço aos anseios do cliente é um fundamento de qualidade perfeitamente aplicável aos diversos serviços de assistência à saúde ${ }^{(24)}$. O aumento da complexidade desses serviços, impulsionado pela demanda tecnológica e pela explosão de novos conhecimentos, acrescidas do aumento da expectativa de vida e do maior número de pacientes portadores de doenças crônicas, vem onerando o gasto em saúde, de modo que o desafio atual desse setor é prestar atendimento humanizado, com alta produtividade e baixo custo. É o que se espera como resultado de programas de qualidade ${ }^{(7,28)}$.

A prestação de serviço em saúde tem implícito dois componentes básicos da qualidade: o operacional, que corresponde ao processo propriamente dito, e a percepção, ou como os clientes percebem o tipo de serviço oferecido ${ }^{(23)}$. Esses componentes podem ser medidos por meio de indicadores da qualidade, e o reconhecimento é obtido pelos processos de certificação ou acreditação.

Os indicadores permitem comparações internas e externas, com outros serviços de mesmas características. São denominados, na gestão da qualidade, itens de controle. Atualmente, a prática de benchmarking possibilita a 
avaliação do desempenho de todos os processos de determinado serviço, comparando seus dados com serviços de referência ${ }^{(35)}$.

Benchmarking foi definido por Bittar como o ato de comparar sistematicamente informações ou, ainda, um padrão de referência pelo qual outros podem ser medidos ou julgados. Pode ser classificado como: interno, quando a comparação ocorre por processos semelhantes entre setores de uma mesma instituição; funcional, se a comparação ocorre entre instituições semelhantes, mas que atuam em mercados distintos; e competitivo, forma mais utilizada, que ocorre a partir da comparação de processos semelhantes entre concorrentes diretos ${ }^{(6)}$.

A certificação atesta que determinados produtos, processos ou serviços são realizados ou cumpridos de acordo com requisitos especificados, como é o caso das normas da International Organization for Standardization (ISO). Já na acreditação, os procedimentos são avaliados com o intuito de verificar sua adequação aos serviços que estão sendo oferecidos, além do cumprimento dos requisitos exigidos em uma certificação. Por exemplo, a acreditação da Organização Nacional de Acreditação (ONA), da Joint Commission on Accreditation of Healthcare Organizations (JCAHO) e do Programa de Acreditação de Laboratórios Clínicos (PALC) da Sociedade Brasileira de Patologia Clínica/ Medicina Laboratorial (SBPC/ML) $)^{(5,23)}$.

A história da acreditação na saúde iniciou-se no século passado, quando o Colégio Americano de Cirurgiões (CAC), em 1924, estabeleceu o Programa de Padronização Hospitalar (PPH), no qual se definiam os requisitos essenciais para a garantia da qualidade da assistência. O PPH descrevia a criação e a organização do corpo clínico e definia o exercício da profissão médica, o preenchimento de prontuário com histórico, os exames e as condições de alta e a existência de recursos diagnósticos e terapêuticos.

No início da década de 1950, a Comissão Conjunta de Acreditação dos Hospitais (CCAH), formada por CAC, Associação Médica Americana, Associação Médica Canadense, Colégio Americano de Clínicos e Associação Americana de Hospitais, criou o programa de acreditação Joint Commision on Acreditation of Hospitals, atualmente chamado JCAHO. A Joint Commission, que inicialmente teve papel fundamental na divulgação da qualidade na cultura médico-hospitalar, tem buscado, desde a década de 1970, com a publicação do manual Accreditation Manual for Hospital, melhorias nos processos hospitalares, bem como nos resultados da assistência, utilizando indicadores de desempenho. Recentemente, tem assumido o papel de educação com monitoramento, publicando uma série de documentos, como normas, padrões e recomendações ${ }^{(10)}$.

No Brasil, a preocupação com a qualidade na área da saúde advém da década de 1930, com a criação da Ficha de Inquérito Hospitalar, por Odair Pedroso, em São Paulo, para a Comissão de Assistência Hospitalar do Ministério da Saúde (MS) ${ }^{(10)}$. Nela, os padrões mínimos de organização hospitalar incluíam corpo clínico organizado, corpo administrativo e de enfermagem, serviços radiológico e fisioterápico, laboratório clínico, necrotério, farmácia e serviços auxiliares (cozinha, lavanderia e desinfecção).

Os programas de acreditação têm início apenas no fim da década de 1980, quando a Organização Mundial da Saúde (OMS) e a Organização Pan-Americana da Saúde (OPAS) elaboraram o manual de padrões de acreditação para a América Latina. Em 1997, o MS decide estabelecer uma comissão nacional de especialistas para o desenvolvimento do modelo brasileiro de acreditação, o qual foi oficialmente lançado em 1998, no Congresso Internacional de Qualidade na Assistência à Saúde, em Budapeste ${ }^{(2,10)}$.

Em 1999, foi criada a ONA, cujo principal objetivo é a implementação nacional de um processo permanente de melhoria da qualidade da assistência à saúde, estimulando os serviços a atingirem padrões mais elevados de qualidade (www.ona.org.br). Em 2001/02, a Agência Nacional de Vigilância Sanitária (ANVISA) reconheceu oficialmente o Sistema Brasileiro de Acreditação por meio da Resolução ${ }^{\circ}$ 921/02 e firmou um convênio com a ONA para cooperação técnica e treinamento de pessoal, o qual contou com a participação, entre outras, da SBPC/ML ${ }^{(2,42)}$.

A SBPC/ML teve papel fundamental na história da qualidade e da acreditação laboratoriais, visto que, em sua fundação, em 1944, já possuía em seu estatuto, como um dos objetivos, o estabelecimento de padrões para a realização dos diferentes exames laboratoriais. No decorrer da década de 1970, propôs revisar e adaptar à realidade brasileira as práticas do Colégio Americano de Patologistas (CAP), por meio da Revista Brasileira de Patologia Clínica, publicação da própria SBPC/ML. No ano de 1977, em contrato com a Control-Lab, a SBPC/ML pôde lançar o programa de controle de qualidade interno e externo, inédito no país, intitulado Programa de Excelência de Laboratórios Médicos (PELM), e, em 1998, criou o PALC, os quais foram revisados e atualizados nos anos 2004, 2007 e 2010. O PALC permite aos laboratórios brasileiros um caminho para a melhoria contínua da qualidade, principalmente pelas auditorias realizadas por pares, ou seja, por laboratoristas, propiciando oportunidades de trocas de conhecimentos técnicos entre 
auditores e auditados ${ }^{(42)}$. Mais recentemente, novamente em parceria com a Control-Lab, a SPBC/ML disponibilizou o Programa de Indicadores Laboratoriais, permitindo aos laboratórios clínicos brasileiros a possibilidade da padronização de seus indicadores, bem como a comparabilidade dos mesmos (www.sbpc.org.br/institucional/historia) ${ }^{(43)}$.

Segundo Plebani, a busca de acreditações é primordial para a melhoria dos serviços laboratoriais, bem como a necessidade de um consenso internacional. Esse movimento foi iniciado a partir da publicação das normas ISO 9001, ISO/IEC 15189:2003 e do programa de acreditação do CAP. No Brasil, Galoro et al. relataram o Programa de Indicadores Laboratoriais (Control-Lab SBPC/ML), iniciado em 2006, como exemplo de um modelo brasileiro de benchmarking na área laboratorial| ${ }^{(11,28)}$.

\section{Qualidade no laboratório clínico}

O constante progresso tecnológico na área laboratorial tem possibilitado a ampliação do número e dos tipos de analitos passíveis de análise, aumentando, significativamente, a importância do laboratório na decisão médica e na tomada de condutas terapêuticas ${ }^{(29)}$. Segundo Westgard e Darcy, os resultados das análises laboratoriais são responsáveis por $65 \%$ a $75 \%$ das informações pertinentes à decisão médica ${ }^{(46)}$.

A medicina laboratorial pode ser considerada setor pioneiro na área médica a promover e introduzir os conceitos da qualidade. Na década de 1960, Barnett e Tonks iniciaram estudos sobre variabilidade biológica, os quais foram aprimorados por Harris e Fraser nas décadas subsequentes. Nos anos 1990, houve um consenso sobre os objetivos da qualidade e suas especificações no ambiente do laboratório clínico ${ }^{(46)}$. Assim, foram definidos os conceitos de controle da qualidade, garantia da qualidade e gestão total da qualidade.

A evolução tecnológica foi uma das principais alavancas que permitiu a implantação dos modernos conceitos da qualidade no laboratório clínico. No entanto, as novas práticas resultaram no aumento do custo global de todo o processo laboratorial, nem sempre acompanhado do aumento na remuneração pelas fontes pagadoras. Ao contrário, os laboratórios clínicos, particularmente no Brasil, passaram a sofrer forte pressão dos provedores de serviço de saúde suplementar, no sentido de diminuir drasticamente os custos de execução dos exames ${ }^{(25)}$.

Para Plebani, uma das consequências da elevação do custo laboratorial refere-se a não adequação do mesmo ao objetivo a que se destina. Um exame é apropriado quando é efetivo e claramente indicado, não custoso e disponível para a população apropriada. Na solicitação de um exame, é necessária a avaliação de seu custo/benefício, ou seja, se o teste trará benefícios para diagnóstico, prognóstico ou tratamento. Caso contrário, o exame pode ter um custo desnecessário, além de aumentar o risco de resultados falsos positivos, desencadear outras investigações e gerar modificações errôneas na terapia, atraso diagnóstico ou aumento da permanência hospitalar ${ }^{(28)}$.

Segundo Ismail, a liberação de um laudo incorreto pode desencadear aumento de consultas médicas e testes laboratoriais e de imagem, elevando ainda mais o custo dos serviços de saúde ${ }^{(19)}$.

A busca de melhoria contínua exigiu, em um primeiro momento, a análise minuciosa dos diferentes processos envolvidos na realização do exame laboratorial, incluindo aspectos técnicos, organizacionais e administrativos, além de identificar desvios e propor oportunidades de melhoria.

Para Plebani, o erro laboratorial é definido como uma falha ocorrida em qualquer parte do ciclo laboratorial, ou seja, desde a solicitação médica até a interpretação e a reação do médico diante do resultado reportado, ou qualquer defeito na realização do teste que gere um resultado inapropriado ou uma interpretação equivocada ${ }^{(31)}$.

Segundo Hollensead, o processo que culmina na execução de um exame laboratorial inicia-se na avaliação clínica, no estabelecimento de uma hipótese diagnóstica e na solicitação de exames dirigida para os diagnósticos aventados. O processo final se concretiza por meio da utilização, pelo médico, da informação gerada pelo laboratório(16).

As diversas etapas de execução de um exame são divididas, classicamente, em três fases: pré-analítica, analítica e pós-analítica(20).

Atualmente, os termos fase pré-pré-analítica e fase póspós-analítica foram introduzidos para definir as etapas de execução de um exame que independem propriamente do laboratório. A pré-pré-analítica corresponde à seleção, pelo clínico, de exames apropriados ao diagnóstico a que se pretende e à solicitação dos mesmos. Coleta, transporte e recepção das amostras adequadas ao exame, quando não são de responsabilidade do laboratório, também podem estar inclusos nessa fase. A pós-pós-analítica refere-se à interpretação do resultado pelo clínico ${ }^{(30,32,33)}$.

Desse modo, o sistema da qualidade do laboratório requer disciplina e organização em todas as etapas dos diferentes processos. Nesse contexto, os indicadores 
laboratoriais permitem avaliar a eficácia e a eficiência das diferentes etapas de execução do exame laboratorial.

\section{Indicadores laboratoriais}

Pode-se definir indicador como uma informação de natureza qualitativa ou quantitativa, associada a um evento, processo ou resultado, sendo possível avaliar as mudanças durante o tempo e verificar ou definir objetivos ou utilizá-lo para a tomada de decisões ou escolhas ${ }^{(32)}$.

Ricós define indicadores laboratoriais como medidas numéricas de erros ou falhas de determinado processo em relação a seu número total (acertos e erros). São especificações da qualidade, pois o desempenho de um processo é considerado satisfatório se estiver nos limites estabelecidos nos indicadores. Seu objetivo não é prover respostas, mas indicar problemas potenciais que necessitam de ações preventivas ${ }^{(35)}$.

A definição do número e dos tipos de indicadores costuma ter como base a complexidade e o tamanho da organização, assim como a missão e os objetivos do serviço(37). A falta de um padrão internacional, ou mesmo nacional, dificulta a definição de metas ou objetivos, assim como a prática de benchmarking, visto que um mesmo indicador pode diferir no modo de reportar os dados, na coleta dos mesmos e na metodologia utilizada para expressar o indicador (percentual ou números absolutos).

Algumas publicações relatam os indicadores mais comumente utilizados pelos laboratórios clínicos e seus respectivos limites de aceitabilidade ${ }^{(4,16,28,33,35,40)}$. Esses dados possibilitam que outros laboratórios clínicos monitorem seus processos em busca da melhoria contínua.

Alguns aspectos necessitam de maior atenção na fase pré-analítica, conforme descritos a seguir:

- orientações acerca do preparo adequado para a coleta;

- identificação correta do paciente;

- informações relevantes, como idade, sexo, raça, uso de medicamentos, entre outros;

- coleta, identificação e transporte da amostra biológica;

- processamento da amostra (centrifugação e aliquotagem).

Estima-se entre $46 \%$ e $68 \%$ o percentual de erros laboratoriais referentes a problemas ocorridos durante a fase pré-analítica. Segundo Plebani, entre os erros observados na fase pré-analítica destacam-se: identificação incorreta, amostra coletada erroneamente ou em volume insuficiente e condição de transporte ou conservação inadequada. São relatados, ainda, problemas de centrifugação, aliquotagem e identificação das alíquotas como erros pré-analíticos ${ }^{(33)}$.

Além disso, a escolha inapropriada de testes laboratoriais ou de seus painéis também pode ser considerado um erro pré-analítico ${ }^{(31)}$.

Indicadores mais comumente citados na literatura, associados a essa fase, referem-se a índices de recoleta, relacionados com falhas anteriormente mencionadas ${ }^{(39)}$. Outros exemplos são:

- erros na abertura de cadastro (identificação equivocada do paciente ou do médico e erro no cadastro dos exames);

- amostras solicitadas e não coletadas;

- falhas na coleta (coleta de tubo errado, hemólise, amostras coaguladas e razão inadequada de sangue/anticoagulante);

- problemas no transporte das amostras.

A fase analítica corresponde à etapa de execução do teste laboratorial, a qual pode ser monitorada pelo controle interno da qualidade (CIQ) e pelos ensaios de proficiência ou avaliação externa da qualidade (AEQ). CIQ e AEQ são ferramentas utilizadas na prática laboratorial para assegurar seus serviços ou, mais especificamente, o processo de realização dos testes ${ }^{(3,37)}$. Controles internos que se assemelham às amostras biológicas são preparados e utilizados em conjunto com estas, com a finalidade principal de monitorar a estabilidade e a reprodutibilidade do sistema analítico durante todas as etapas de sua execução ${ }^{(34)}$. As amostrascontrole possuem valores conhecidos e seu monitoramento é realizado por meio das análises estatísticas, como média, desvio padrão e coeficiente de variação. O gráfico de LeveyJennings e as regras múltiplas de Westgard são ferramentas mundialmente difundidas na avaliação do $\mathrm{CIQ}$, sendo utilizadas há pelo menos 20 anos. O CIQ permite, sobretudo, avaliação do erro aleatório de uma análise ${ }^{(45)}$.

O AEQ tem por finalidade avaliar o desempenho dos sistemas analíticos por meio de ensaios de proficiência e realizar comparações interlaboratoriais ${ }^{(34)}$. A simples participação em um programa de ensaio de proficiência não garante o bom desempenho dos testes avaliados. Os dados fornecidos devem ser utilizados para comparação com resultados das diversas rodadas subsequentes. É possível calcular o erro total permitido para cada analito, utilizando-se dados de variabilidade biológica ${ }^{(36,37)}$. O erro total é, por definição, a soma dos erros sistemático e aleatório (ou variação aleatória) que podem ocorrer em uma análise, como exemplificado na Figura. 


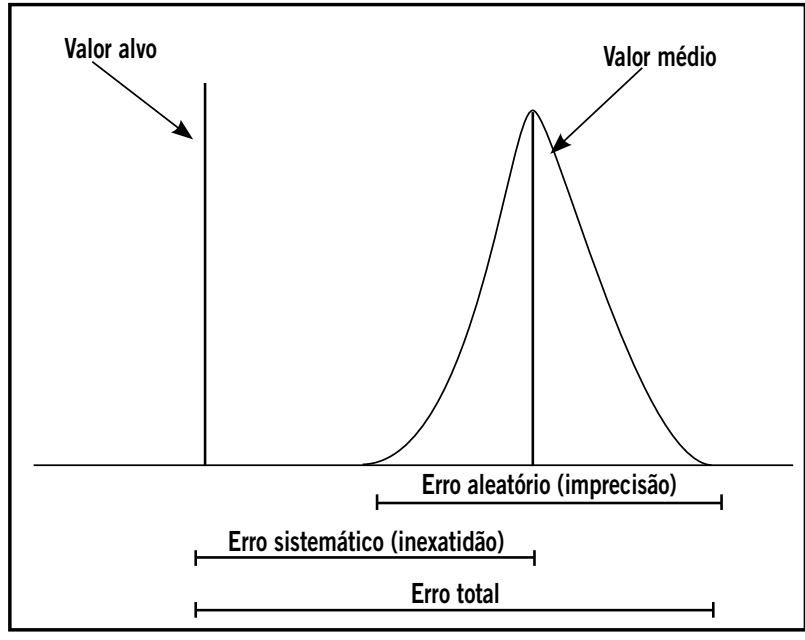

Figura 1 - Representação esquemática do erro total

Nos EUA, o CAP disponibiliza os programas Q-Probe e Q-Traks para avaliação externa da qualidade. No Brasil, os ensaios de proficiência da Control-Lab, vinculados à SBPC/ML, e o Programa Nacional de Controle de Qualidade (PNCQ) da Sociedade Brasileira de Análises Clínicas (SBAC) são os mais difundidos (www.controllab.com.br; www.pncq.org.br).

Recentemente, Westgard demonstrou que, tendo como base a escala sigma, resultados das fases analíticas de ensaios simples e bem estabelecidos, como a dosagem de colesterol total, cálcio, glicose e hemoglobina glicada, não são totalmente satisfatórios. Foram analisados dados de um ensaio de proficiência de aproximadamente 9 mil participantes para esses analitos e concluiu-se que, na métrica sigma, os melhores resultados variam em torno de três a quatro sigmas, bem distantes dos seis sigmas idealizados na área industrial(46).

São poucos os indicadores da fase analítica mencionados na literatura, sendo que estes se baseiam, principalmente, no número de resultados inaceitáveis de $\mathrm{CIQ}$ e $\mathrm{AEQ}$, conforme descrito na Tabela 1 $\mathbf{1}^{(9,33,35)}$. Segundo Plebani, a dificuldade de relatar os erros da fase analítica ocorre pela dificuldade de observá-los, visto que aproximadamente $75 \%$ deles resultam em valores na faixa de referência e $12,5 \%$ produzem resultados totalmente incoerentes, os quais são prontamente corrigidos. Desse modo, 12,5\% são os erros que podem afetar o cuidado ao paciente. Estes podem ser minimizados com treinamento e qualificação da equipe profissional do laboratório e adoção de condutas de identificação e correção de falhas de CIQ e AEQ ${ }^{(18,31)}$.

A fase pós-analítica, por sua vez, tem início no ambiente do laboratório clínico e envolve os processos de validação e liberação de laudos e se encerra após o médico receber

\section{Exemplos de indicadores nas fases}

Tabela 1 pré-analítica, analítica e pós-analítica

\begin{tabular}{ll}
\hline Fase de processos & Indicadores \\
laboratoriais & - Recoleta \\
& - Erros na abertura de cadastro \\
& - Amostras solicitadas e não \\
& coletadas \\
Pré-analítica & - Falhas na coleta \\
& - Problemas no transporte das \\
& amostras \\
& - Percentual de resultados \\
& inaceitáveis no CIQ \\
Analítica & Percentual de resultados \\
& inaceitáveis no AEQ \\
& - Sucesso na comunicação de \\
& valores críticos \\
& - Percentual de resultados \\
& liberados no prazo \\
& - Intercorrências na liberação de \\
& resultados \\
Pós-analítica & Exames liberados e não \\
& solicitados \\
& - Exames solicitados e não \\
& liberados \\
& Percentual de laudos retificados \\
\hline
\end{tabular}

$C I Q$ : controle interno da qualidade; $A E Q$ : avaliação externa da qualidade.

o laudo final, seguido de sua interpretação e tomada de decisão perante o resultado reportado(31).

Erros frequentemente associados a essa etapa correspondem às falhas na liberação dos resultados, por erros de transcrição ou digitação ou pelo não cumprimento do prazo de entrega. $O$ desenvolvimento da tecnologia de informação (TI) aplicada ao setor laboratorial tem contribuído para a diminuição dos erros de transcrições de resultados, principalmente após o advento dos sistemas de interfaceamento, os quais possibilitam a transmissão das informações diretamente do equipamento automatizado de análise para o sistema de informação laboratorial (SIL) ${ }^{(15,31)}$. A identificação das amostras por meio das etiquetas de código de barras também tem sido fundamental na busca de melhoria da qualidade e redução dos $\operatorname{erros}^{(43)}$.

Outros processos vinculados à fase pós-analítica são relacionados com as informações adicionais contidas em um laudo laboratorial, além do resultado. Trata-se dos valores de referência e comentários que auxiliam na interpretação clínica. Valores de referência de determinado analito são, 
na maior parte das vezes, transcritos das instruções do fabricante do conjunto diagnóstico e podem não representar adequadamente a população em estudo(14). Do mesmo modo, informações quanto à sensibilidade do método utilizado, coeficiente de variação, entre outros, devem ser acrescentados aos laudos com o objetivo de informar as peculiaridades metodológicas das análises. Falhas nessas informações devem ser consideradas erros laboratoriais, pois influenciam diretamente a interpretação clínica ${ }^{(41)}$.

O tempo total de liberação do resultado também é um quesito a ser verificado na garantia de qualidade do laboratório, principalmente para exames cujo tempo de liberação influencia diretamente a decisão clínica ou quando há resultados críticos que devem ser comunicados com rapidez. A falta de notificação imediata de valores críticos pode ser tão negativa quanto a liberação de resultados inadequados. Os erros recorrentes dessa fase do ciclo laboratorial giram em torno de $18 \%$ a $47 \%(33,35)$.

São exemplos de indicadores da fase pós-analítica: sucesso na comunicação de valores críticos, percentagem de resultados liberados no prazo e intercorrências na liberação de resultados, exames liberados e não solicitados, exames solicitados e não liberados e porcentagem de laudos retificados ${ }^{(33,35)}$.

Os indicadores laboratoriais auxiliam na padronização e na definição das especificações da qualidade para o processo de realização de um exame. Ainda não há consenso acerca dos melhores indicadores para as fases analíticas e extra-analíticas, bem como seus respectivos limites de aceitabilidade, uma vez que há grande variabilidade nos processos, principalmente nas fases pré e pós-analíticas, dos diversos laboratórios clínicos. Desse modo, uma alternativa muito aplicada atualmente refere-se à prática de benchmarking, em especial para comparar os indicadores mais conhecidos e utilizados, como índices de recoleta, amostras coaguladas e hemolisadas ${ }^{(31,40)}$.

Galoro et al. relataram a dificuldade na padronização e na coleta de dados, assim como a consistência dos dados obtidos para a prática de benchmarking. Os autores sugerem a segregação dos participantes com características semeIhantes em grupos e cita o exemplo brasileiro do Programa de Indicadores Laboratoriais, desenvolvido pela SBPC/ML e pelo Control-Lab(11).

A Tabela 2 descreve indicadores adotados pelo Programa de Indicadores Laboratoriais da SBPC/ML e do Control-Lab.

A International Federation of Clinical Chemistry and Laboratory Medicine (IFCC) criou, recentemente, o projeto Model of Quality Indicator, que se encontrava em fase
Indicadores do Programa de Indicadores

Tabela 2 Laboratoriais da SBPC/ML e do Control-Lab

\begin{tabular}{ll}
\hline Tipo & Indicador \\
& - Exames por paciente \\
& - Públicos atendidos \\
Demográfico & - Sistemática de coleta \\
& - Terceirização \\
& - Ticket médio \\
& - Volume de exames \\
& - Acidente com perfurocortante \\
& - Cliente \\
Processual & - Qualidade de amostras \\
& - Recoleta \\
& - Entrega de laudo \\
& - Despesa com pessoal \\
& - Distribuição de despesas \\
& - Frequência de acidente de trabalho \\
Gestão de & - Glosa \\
recursos & - Informatização \\
& - Pessoal \\
& - Produtividade \\
& - Treinamento \\
\hline
\end{tabular}

experimental em $2009^{(38)}$. O estudo reporta um projeto para padronização de indicadores, em todas as suas fases (definição, coleta, reporte e análise de dados), em nível internacional, visto que normas de acreditação, a exemplo da ISO 15189:2007, recomendam a implantação, o monitoramento e a avaliação sistemática dos processos laboratoriais para contribuição dos laboratórios ao cuidado aos pacientes, sendo a maneira mais adequada ao monitoramento o uso de indicadores e sua comparabilidade com serviços semelhantes ou pares.

A Tabela 3 descreve alguns indicadores descritos na literatura com os níveis observados pelos diferentes autores, bem como identifica aqueles adotados pelo programa de indicadores da SBPC/ML e do Control-Lab.

Atualmente, a melhoria da qualidade faz parte da rotina dos profissionais de laboratório, mas ela não é possível se não for medida e comparada com referenciais.

\section{Conclusão}

Quando se buscam informações sobre indicadores de qualidade na área da medicina laboratorial, são poucos os artigos encontrados na literatura, pois é um tema ainda recente para a área, principalmente quando se fala da comparabilidade de dados ou benchmarking. 


\begin{tabular}{|c|c|c|}
\hline \multicolumn{3}{|c|}{$\begin{array}{l}\text { Exemplos de indicadores da qualidade descritos na literatura com os respectivos índices dos } \\
\text { diferentes autores }\end{array}$} \\
\hline Indicador da qualidade & $\begin{array}{l}\text { Índices descritos } \\
\text { pelos autores (\%) }\end{array}$ & $\begin{array}{l}\text { Adotado pelo Programa de Indicadores } \\
\text { Laboratoriais (SBPC/ML e Control-Lab)? }\end{array}$ \\
\hline Erros de cadastro de exames & $\begin{array}{c}0,30^{(26)} \\
0,31^{(33,35)} \\
1 \text { a } 2 \text { internados e } 0,2 \text { a } 6 \\
\text { ambulatório(22) } \\
12,9^{(32)} \\
4,8^{(44)} \\
4,1^{(21)}\end{array}$ & Não \\
\hline Recoleta & $\begin{array}{l}1,9^{(26)} \\
2^{(33,35)}\end{array}$ & Sim \\
\hline $\begin{array}{l}\text { Resultados inadequados em teste de } \\
\text { proficiência }\end{array}$ & $\begin{array}{c}0,9^{(26)} \\
1,4^{(33,35)} \\
0,9 \text { e } 1,7^{(17)} \\
0,8^{(21)}\end{array}$ & Não \\
\hline Coleta em recipiente impróprio & $\begin{array}{c}0,002^{(35)} \\
0,0015^{(33)} \\
0,02 \text { (desejável) a }^{\text {0,2(mínimo) }} \text { (38) }^{(38} \\
8,1^{(8)} \\
0,04^{(39)} \\
5^{(21)}\end{array}$ & Sim \\
\hline Amostras coaguladas em hematologia & $\begin{array}{c}0,20^{(33,35)} \\
0,25^{(39)}\end{array}$ & Sim \\
\hline Laudos retificados & $0,05^{(26,35)}$ & Sim \\
\hline Atraso na entrega de resultados & $\begin{array}{l}11^{(33,35)} \\
0,7^{(21)}\end{array}$ & Sim \\
\hline Falha na comunicação de resultados críticos & $\begin{array}{c}21,3^{(33,35)} \\
5^{(17)} \\
3,5^{(27)} \\
0,5^{(21)}\end{array}$ & Sim \\
\hline
\end{tabular}

De modo geral, nota-se grande heterogeneidade nos valores obtidos para um mesmo indicador nas publicações relacionadas, denotando a inexistência de um padrão mundialmente aceito.

Os artigos de Plebani(33) e Ricós ${ }^{(35)}$ são compilados de dados da literatura e compreendem maior número de indicadores subdivididos nas fases pré-analítica, analítica e pós-analítica. Os demais artigos relatam experiências de serviços individuais e, geralmente, o comparativo ocorre no próprio serviço, observando-se melhorias na série histórica.

Nesse contexto, o programa de indicadores da SBPC/ML e do Control-Lab representa um grande avanço na medicina laboratorial brasileira por se tratar de um processo de padronização e comparabilidade de indicadores entre os laboratórios clínicos brasileiros. Deve-se destacar também o projeto Model of Quality Indicator, da IFCC, iniciado em 2009. Apesar dos avanços, ainda não há consenso sobre os melhores indicadores a serem adotados pelos laboratórios.

A busca da padronização dos indicadores da qualidade nem sempre é uma tarefa fácil quando se envolvem diversos laboratórios clínicos, cada qual com características distintas, mas que procuram trocar experiências com seus pares nos programas de benchmarking. Cabe aos gestores o desafio de ampliar os conhecimentos, envolver e treinar a equipe de colaboradores, visando difundir os conceitos e a importância dos indicadores na gestão dos processos. 


\section{Referências}

1. ALGARTE, W.; QUINTANILHA, D. A história da qualidade e o programa brasileiro da qualidade e produtividade. Rio de Janeiro: INMETRO/SENAI, 2000.

2. ANVISA. Acreditação: a busca pela qualidade nos serviços de saúde. Rev Saúde Pública, v. 38, n. 2, p. 335-6, 2004.

3. BERLITZ, F. A.; HAUSSEN, M. L. Seis sigma no laboratório clínico: impacto na gestão de performance analítica dos processos técnicos. J Bras Patol Med Lab, v. 41, n. 5, p. 301-12, 2005.

4. BONINI, P. et al. Errors in laboratory medicine. Clin Chem, v. 48, n. 5, p. 691-98, 2002.

5. BITTAR, O. J. N. V. Gestão de processos e certificação para qualidade em saúde. Rev Assoc Med Bras, v. 46, ก. 1, 2000.

6. BITTAR, O. J. N. V. Indicadores de qualidade e quantidade em saúde. Rev Adm Saúde, v. 3, n. 12, 2001.

7. BITTAR, O. J. N. V. Indicadores de qualidade e quantidade em saúde - parte II. Rev Adm Saúde, v. 6, n. 22, 2004.

8. CARRARO, P.; PLEBANI, M. Errors in a stat laboratory: changes in type and frequency since 1996. Clin Chem, v. 53, n. 7, p. 1-5, 2007

9. CARRARO, P., PLEBANI, M. Errors in a stat laboratory: types and frequencies 10 years later. Clin Chem, v. 53, n. 7, p. 1338-42, 2007.

10. FELDMAN, L. B. et al. História da evolução da qualidade hospitalar: dos padrões à acreditação. Acta Paul Enferm, v. 18, n. 2, p. 213-9, 2005.

11. GALORO, C. A. O. et al. Applicability and potential benefits of benchmarking in Brazilian clinical laboratory services. Benchmark Int J, v. 16, n. 6, p. 817-30, 2009.

12. GOMES, P. J. P. A evolução do conceito de qualidade: dos bens manufaturados aos serviços de informação. Cadernos Bad, v. 2, p. 6-18, 2004.

13. GURGEL JUNIOR, G. D.; VIEIRA, M. M. F. Qualidade total e administração hospitalar: explorando disjunções conceituais. Ciência \& Saúde Coletiva, v. 7, n. 2, p. 325-34, 2002.

14. GROSSI, E. et al. The REALAB project: a new method for the formulation of reference intervals base don current data. Clin Chem, v. 51, n. 7, p. 1232-40, 2005.

15. HILT, L. et al. Project Control for Laboratory Automation Outsourced to consultants: a 10-step process to optimize the effectiveness of custom information technology development. J Assoc Lab Autom, v. 8, n. 1, p. 31-7, 2003

16. HOLLENSEAD, S. C. et al. Errors in pathology and laboratory medicine: consequences and prevention. J Surg Oncol, v. 88, p. 161-81, 2004.

17. HOWANITZ, P. J. Errors in laboratory medicine: practical lessons to improve patient safety. Arch Pathol Lab Med, v. 129, p. 1252-61, 2005.

18. HOWANITZ, P. J. et al. Clinical laboratory quality control: a costly process now out of control. Clin Chim Acta, v. 260, n. 2, p. 163-74, 1997.

19. ISMAIL, A. A. A. et al. Wrong biochemistry results: two cases reports and observational study in 5310 patients on potentially misleading thyroid-stimulating hormone and gonadotropin immunoassay results. Clin Chem, v. 48, n. 11, p. 2023-9, 2002.

20. KANASHIRO-CUSSIOL, A. et al. Changes in costs over time at a medium-sized clinical laboratory. Lab Medicine, v. 41, n. 3, p. 145-6, 2010.

21. KIRCHNER, M. J. et al. Quality indicators and specifications for key processes in clinical laboratories: a preliminary experience. Clin Chem Lab Med, v. 45, n. 5, p. 672-7, 2007.

22. LIPPI, G. et al. Causes, consequences, detection and prevention of identification errors in laboratory diagnostics. Clin Chem Lab Med, v. 47, n. 2, p. 143-53, 2009.

23. MALIK, A. M.; SCHIESARI, L. M. C. Qualidade na gestão local de serviços e ações de saúde. Núcleo da Assistência Médico-Hospitalar; Editora Fundação Peirópolis, 1998.

24. MENDES, M. E. Avaliação da implantação de um sistema de qualidade em um laboratório clínico público. 1998. Tese (Doutoramento) - Faculdade de Medicina, Universidade de São Paulo, São Paulo, 1998.

25. MENDES, M. E. et al. Gestão por processos no laboratório clínico: uma abordagem prática. Editora EPR, 2007.

26. NEVALAINEN, D. et al. Evaluating laboratory performance on quality indicators with the six sigma scale. Arch Pathol Lab Med, v. 124, p. 516-9, 2000.

27. PIVA, E. et al. Evaluation of effectiveness of a computerized notification system for reporting critical values. Am J Clin Pathol, v. 131, p.432-41, 2009.

28. PLEBANI, M. Appropriateness in programs for continuous quality improvement in clinical laboratories. Clin Chim Acta, v. 333, p. 131-9, 2003.

29. PLEBANI, M. Charting the course of medical laboratories in a changing environment. Clin Chim Acta, v. 319, n. 2, p. 87-100, 2002.

30. PLEBANI, M. Errors in clinical laboratories or errors in laboratory medicine? Clin Chem Lab Med, v. 44, n. 6, p. 750-9, 2006.

31. PLEBANI, M. Errors in laboratory medicine and patient safety: the road ahead. Clin Chem Lab Med, v. 45, n. 6, p. 700-7, 2007.

32. PLEBANI, M. Exploring the iceberg of errors in laboratory medicine. Clin Chim Acta, v. 404, p. 16-23, 2009.

33. PLEBANI, M. et al. Laboratory network of excellence: enhancing patient safety and service effectiveness. Clin Chem Lab Med, v. 44, n. 2, p. 150-60, 2006.

34. ANVISA. Resolução da Diretoria Colegiada, RDC 302, de 13 de outubro de 2005. ANVISA. Disponível em: $<$ http://e-legis.anvisa.gov.br/leisref/public/showAct. php?id=19176\&word=>. Acesso em: 23 abr. 2011.

35. RICÓS, C. et al. Quality indicators and specifications for the extra-analytical phases in clinical laboratory management. Clin Chem Lab Med, v. 42, n. 6, p. 578-82, 2004.

36. SCIACOVELLI, L. et al. External quality assessment: an effective tool for clinical governance in laboratory medicine. Clin Chem Lab Med, v. 44, n. 6, p. 740-9, 2006. 
37. SCIACOVELLI, L. et al. Risk management in laboratory medicine: quality assurance programs and professional competence. Clin Chem Lab Med, v. 45, n. 6, p. 756-65, 2007.

38. SCIACOVELLI, L.; PLEBANI, M. The IFCC Working Group on laboratory errors and patient safety. Clin Chim Acta, 2009.

39. SCHULTZ, I. M. et al. Preanalytic error tracking in a laboratory medicine department: results of 1-year experience. Clin Chem, v. 52, n. 7, p. 1442-3, 2006.

40. VALENSTEIN, P. N. et al. Staffing benchmarks for clinical laboratories. Arch Pathol Lab Med, v. 129, p. 467-73, 2005.

41. VALENSTEIN, P. N.; SIROTA, R. L. Identification errors in pathology and laboratory medicine. Clin Lab Med, v. 24, n. 4, p. 979-96, 2004.
42. VIEIRA, L. M. F. Nova era para a acreditação de laboratórios. J Bras Patol Med Lab, v. 41, n. 4, Editorial, 2005.

43. VIEIRA, L. M. F. SBPC/ML: 60 anos. J Bras Patol Med Lab, V. 40, n. 3, Editorial, 2004.

44. WANG, S. et al. Correction of clinical chemistry test results in a laboratory information system. Arch Pathol Lab Med, v. 128, p. 890-2, 2004.

45. WESTGARG, J. O. Abuses, misuses and in-excuses for problems with "Westgard Rules". Disponível em: <http://www.westgard.com/lesson73.htm>. Acesso em: 23 abr. 2011.

46. WESTGARG, J. O.; DARCY, T. The truth about quality: medical usefulness and analytical reliability of laboratory tests. Clin Chim Acta, v. 346, n. 1, p. 3-11, 2004. 